\title{
Sub-GeV dark matter and neutrino searches with Skipper-CCDs: status and prospects.
}

\author{
A. M. Botti, ${ }^{a, b, *}$ M. Cababié, ${ }^{a, b}$ J. Estrada, ${ }^{b}$ G. Fernandez-Moroni, ${ }^{b}$ M. Sofo Haro ${ }^{b, c}$ \\ and J. Tiffenberg ${ }^{b}$ \\ ${ }^{a}$ Department of Physics, FCEN, University of Buenos Aires and IFIBA, CONICET, Buenos Aires, Argentina \\ ${ }^{b}$ Fermi National Accelerator Laboratory, PO Box 500, Batavia IL, 60510, USA \\ ${ }^{b}$ Centro Atómico Bariloche, CNEA/CONICET/IB, Bariloche, Argentina \\ E-mail: abotti@df.uba.ar
}

Thick fully-depleted charge-coupled devices (CCDs) with high-resistivity silicon are used in a wide range of scientific applications, from particle detection to astronomical imaging. Their low noise and high charge collection efficiency allow us to reach unprecedented sensitivity to physical processes with low-energy transfers. The newly-developed Skipper-CCD enhances this sensitivity by reducing the read-out noise reaching a sub-electron resolution. In this work, we summarize the fundamentals of the skipper-CCD operation and the prospects for both sub-GeV dark-matter searches and the detection of coherent elastic neutrino-nucleus scattering. A brief discussion of the challenges associated with the construction of the foreseen detectors with multi-kilogram target mass is also presented.

$37^{\text {th }}$ International Cosmic Ray Conference (ICRC 2021)

July 12 th - 23rd, 2021

Online - Berlin, Germany

${ }^{*}$ Presenter 


\section{Introduction}

Since their invention in 1969 [1], Charge Coupled Devices (CCDs) have been widely used both in scientific and industrial applications [2-4]. With high-resistivity fully-depleted CCDs [5] photon interactions from energies as low as the silicon bandgap can be detected. The number of electron-hole pairs produced in the silicon after an interaction, and subsequently collected, is set by the energy deposited in the bulk. In this sense, CCDs are low-energy calorimeters and have become one of the most promising technology to characterize physic processes with energy transfers bellow $\mathrm{keV}$. However, the sensitivity to small energy deposits has been historically limited by the detector read-out noise, which typically produce an uncertainty in the number of collected charges of a few electrons [6,7].

The implementation of a floating gate $[8,9]$ at the CCD output stage, which allows multiple lectures of the collected charges in a non-disruptive way, has boosted the CCD capabilities by reducing their read-out noise. A sub-electron resolution was achieved with the Skipper-CCDs technology [10] enhancing the detector sensitivity for interactions where only one or two electrons are produced. In addition, by reducing the read-out noise it is also possible to produce the most precise measurements of silicon properties using X-ray interactions with larger energy transfers [11].

Currently, the low-energy threshold of scientific CCDs is being exploited in new-physics and rare-event searches. Light-dark-matter interactions can be detectable by coupling several CCD to reach an active mass and, in turn a total exposure, large enough to set improved limits in the cross-section/mass parameter space [12]. In addition, the implementation of CCDs to detect neutrino interactions [13] has already produced the first results for coherent elastic neutrino-nucleus scatterings $(\mathrm{CE} v \mathrm{NS})$ at nuclear reactors with an energy threshold one order of magnitude lower than the threshold used for the first detection of CE $v$ NS by the COHERENT experiment [14].

An example of a CCD used in dark-matter searches is presented in the left panel of Fig 1. We show a picture of a parasitically produced CCD used for development and testing purposes. The sensor is fabricated with about $10 \mathrm{k} \Omega$ resistivity silicon and has a total active mass of about $0.1 \mathrm{~g}$. The CCD is glued to a silicon substrate and wire-bonded to a flex cable for control and read-out. These CCDs are operated at about $140 \mathrm{~K}$ to reduce the silicon dark-current, being this the expected main source of background for rare-events searches. In this work, we present the Skipper-CCD technology, its performance, and prospects of its implementation in ultra-light dark matter and neutrino detection.

\section{Skipper-CCD working principle}

When a particle impinges on the silicon crystal, energy is transferred to the lattice producing electron-hole pairs; an illustration is presented in the right panel of Fig. 1. Depending on the kind of incident radiation, different scenarios are expected. For a photon, electrons in the silicon valence band are knocked into the conduction band either by Compton scattering or the internal photoelectric effect. For charge leptons, a similar process is expected from the electromagnetic interaction between the impinging particle and the electrons in the lattice, producing tracks across the CCD as the particle passes through. For a dark-matter particle different processes are theorized. Recoils may be produced after a dark matter and electron interaction either through a mediator or 

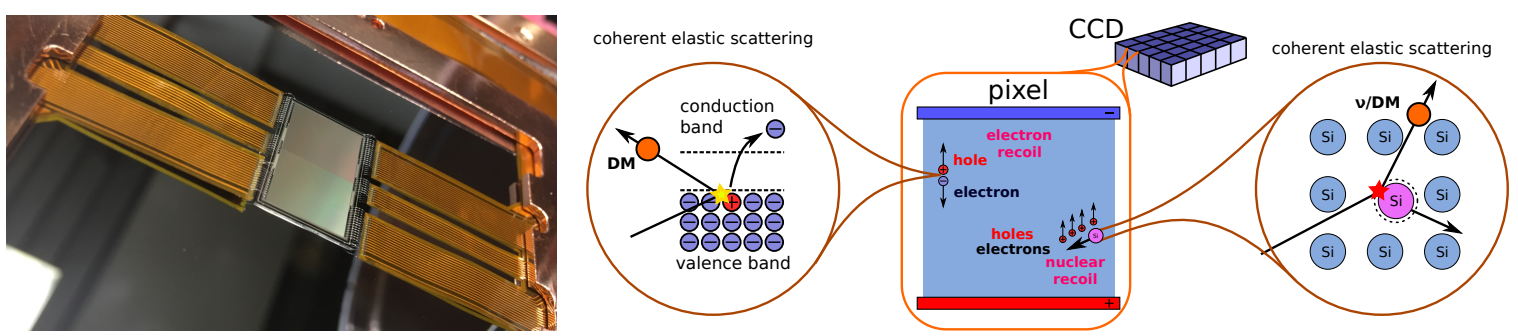

Figure 1: (Left) picture of a CCD use in dark-matter searches. (Right) working principle of CCDs for dark-matter and neutrino detection.

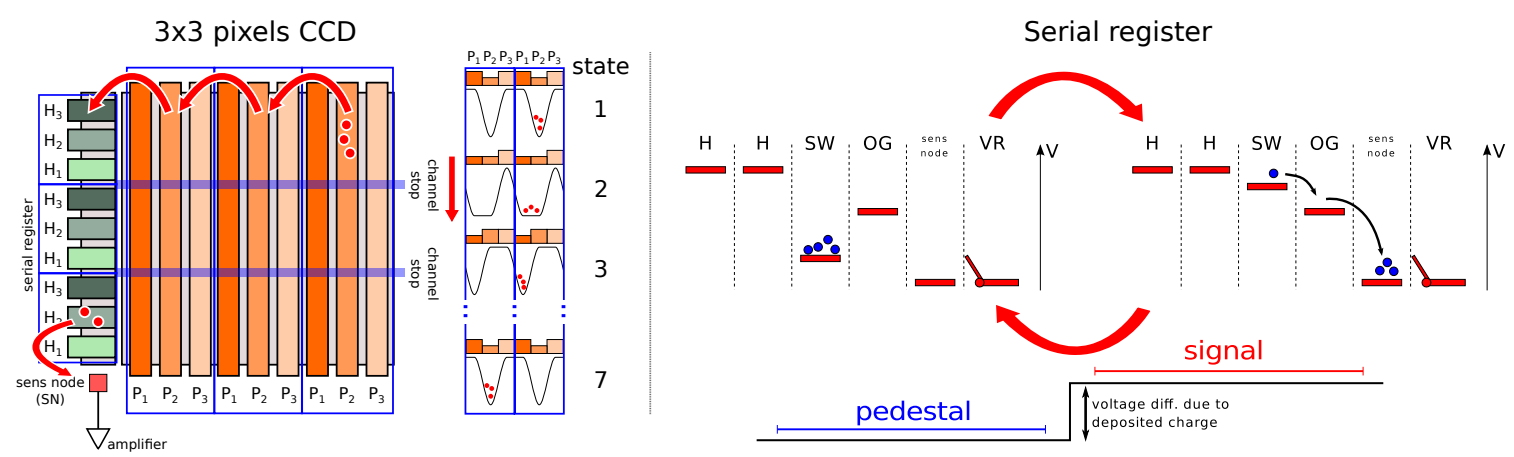

Figure 2: (Left) schematic of the process to transport collected charges to the output stage. (Right) representation of the Skipper-CCD read-out.

direct absorption [15]. Furthermore, a nuclear recoil may be detected as a result of a dark matter and silicon nucleus interacting through a mediator; this same scenario is considered when the impinging radiation consists of neutrinos, where the mediator may be a standard model boson, or even a new particle [16].

Regardless of the process by which the electron-whole pairs are produced, once electrons jump to the conduction band, they are accelerated towards the CCD surface by applying a bias voltage between the CCD terminals. At the surface, charges are transported to the output stage, dubbed serial register, as we illustrate in the left panel of Fig 2 where we present an example of a $3 \times 3$-pixel CCD. Using a series of vertical clocks to set up potential wells along the pixels, we move the charge between the vertical tracks towards the serial register. The time representation of this process is presented as states one to seven in the figure. After reaching the serial register, the charge is transported towards a floating gate, dubbed sense node, with a series of horizontal clocks in the same way as before. It is worth mentioning, that a high transport efficiency is guaranteed and needed in this process to achieve an accurate reconstruction of the energy deposit in the silicon bulk.

In the right panel of Fig. 2, we present a schematic of the serial register for a Skipper-CCD. We use the horizontal clocks to move the charge towards the summing well (SW) gate and then to the sense node through the output gate (OG) [17]. The novelty in the skipper technology is that by implementing a floating gate, we can read the charge in a non-disruptive way and move it back and forward from the sense node to the summing well to obtain multiple measurements of the same charge. Each measurement consists of first integrating the signal in a certain time window when 

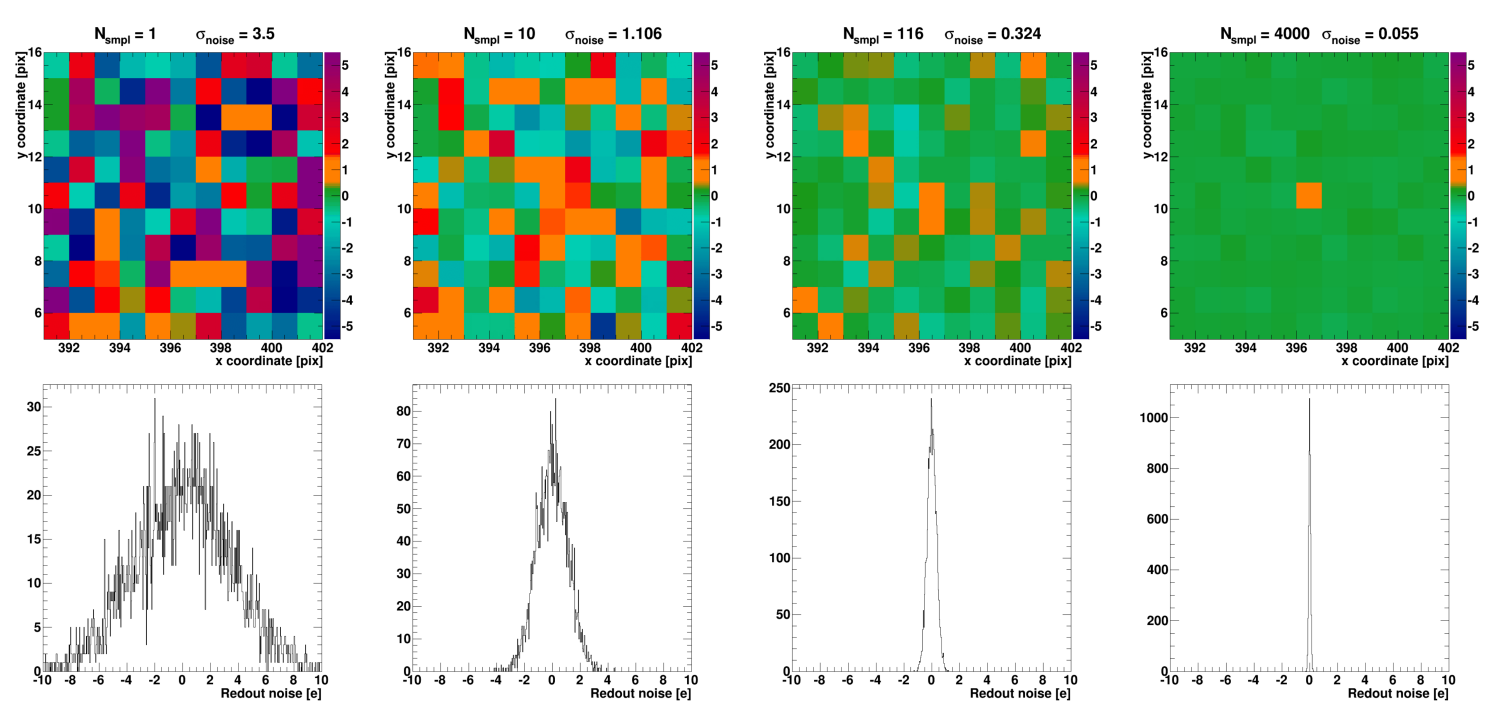

Figure 3: (Top) images obtained with a Skipper-CCD using 1, 10, 116, and 4000 samples. (Bottom) charge distribution of the pixels in the images on the top.

the sense node is empty (left in the schematics) to obtain the signal baseline or pedestal. Then, we move the charges into the sense node (right in the schematics) and repeat the integration to extract the signal. By subtracting the signal and pedestal we obtain a voltage relative to the number of collected charges. This correlated double sampling technique [18] is appropriate to subtract the high-frequency noise since it is averaged to zero at each integration, and the remaining offset is then subtracted. However, it is not enough to remove the noise when $\frac{1}{f}$ is smaller than the integration window, where $f$ is the noise frequency. As we present in the following section, the low-frequency noise can be effectively controlled with the skipper technique.

\section{Performance}

The low-frequency noise is the main contribution to the read-out noise and limits the detector energy resolution: a few electrons for traditional CCDs. This scenario is drastically improved when using Skipper-CCDs to perform several lectures on the output charge. Since for each lecture the noise is mostly uncorrelated and roughly Gaussian, the total read-out noise after averaging all the samples is $\sigma_{N}=\frac{\sigma_{1}}{\sqrt{N}}$, where $\sigma_{1}$ is the noise for one sample and $N$ the number of samples. To illustrate this, we present in the top panels of Fig. 3 cropped images consisting of mainly empty pixels obtained with a skipper-CCD using 1, 10, 116, and 4000 samples. In the bottom panels of Fig. 3 we present the charge distribution in the number of electrons for each image. It is apparent how the read-out noise, estimated as the standard deviation of these distributions, is reduced when the number of samples is increased.

To better illustrate the performance of skipper-CCDs, we present in the left panel of Fig. 4 the read-out noise as a function of the number of samples. The red line represents the theoretical expectation after estimating the noise for one sample. The model describes accurately the data up to about 900 lectures, where a change of regime seems to appear. At this point, the correlated noise between samples starts to dominate and, therefore, stops following the $\frac{1}{\sqrt{N}}$ law. An example image 

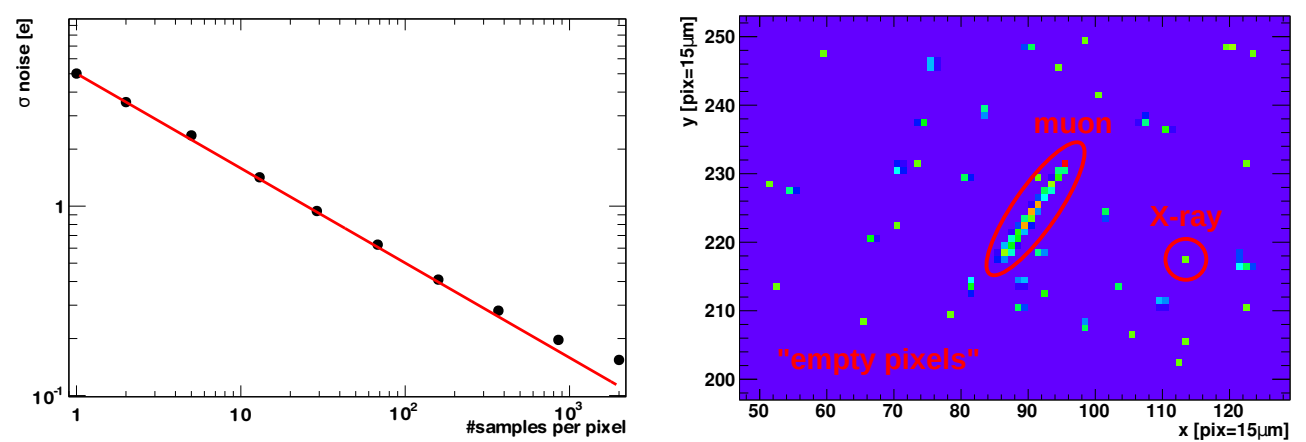

Figure 4: (Left) read-out noise as a function of the number of samples. The red line is a theoretical expectation from the noise for one sample. (Right) example image acquired by averaging 4000 samples.
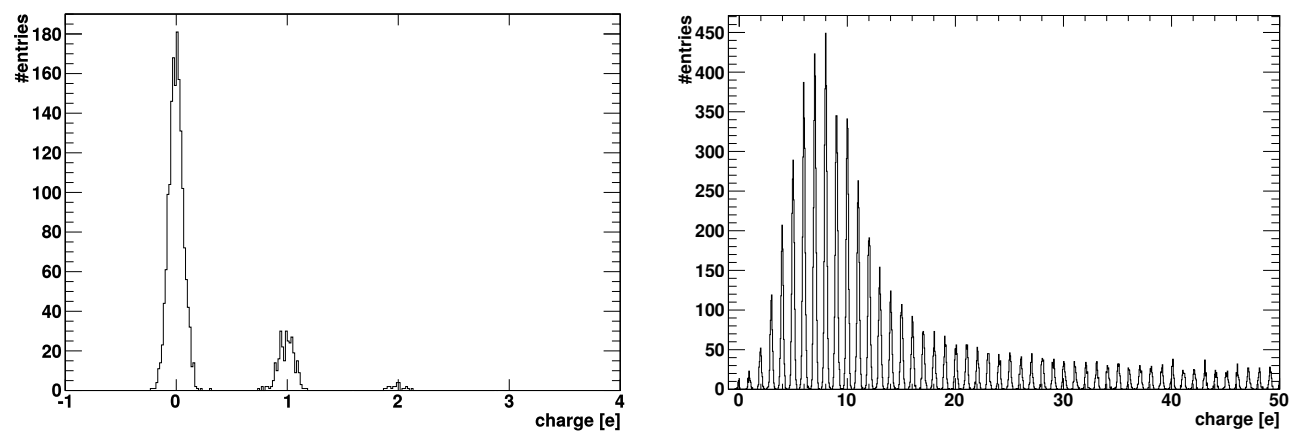

Figure 5: Charge histogram for a non-illuminated CCD (left) and a CCD non-uniformly illuminated with a LED (right).

obtains averaging 4000 samples is shown in the right panel of Fig. 4, where a muon track and several $\mathrm{X}$-rays are distinguishable. The color coding corresponds to the number of electrons measured in each pixel; those with charge are easily identified from those with no charge. Defining the operation point of the CCD results from balancing the noise level and read-out time. Reducing the noise implies increasing the exposure by about $10 \mu s$ per pixel, and in turn, increasing the probability of obtaining dark-current events with more than one electron. Furthermore, in presence of radiation, the exposure needs to be controlled to avoid multiple events hitting the same pixels, producing a spatial pile-up.

In absence of incident radiation, only the detector noise can fill the CCD pixels with charge [19]. We present an example of this scenario in the left panel of Fig. 5, where the pixel charge distribution is shown; the baseline, the one- and two-electrons peaks are observed. This represents the typical scenario on a dark-matter experiment: if dark matter particles interact with the silicon producing two or three electron-whole pairs, an excess on the expected background in these energy channels would be observed. We present a different case in the right panel of Fig. 5, where we illuminated the CCD in a non-uniformly way with a LED. Regardless of the radiation source filling the CCD with charge, each number of collected electrons is distinguishable from its consecutive: the sub-electron resolution of the Skipper-CCD allows us to count with unprecedented precision the number of charges in each pixel. An absolute calibration of the CCD is then possible without depending on a previously calibrated source of light or photo-detector. In rare-events searches, the clear separation between energy channels plays a key role in the identification of a signal between and/or above the 
different backgrounds.

\section{Prospects in dark matter and neutrino detection}

Skipper-CCDs are a promising technology to exploit in rare events and new physics experiments where sensitivity to low-energy processes is required. However, several are the challenges for building a successful setup to this aim; controlling the environmental background has become one of the main difficulties in these experiments. The cosmic radiation leaves tracks in the CCDs that need to be removed from any analysis. Furthermore, when a high number of charges is transferred from a pixel to the serial register, electrons may get caught in silicon impurities or bleed to nearby pixels, and later on, be reconstructed as single- or few-electrons events. Even though this noise is distinguishable and is removed by rejecting areas in the CCD around high-energy events, it reduces drastically the active mass of the detector. In addition, environmental photons and electrons also leave signals in the CCD that maybe be similar to that expected from a dark-matter particle interaction. Deploying the set up in an underground facility is necessary to reduce the cosmic muon flux, and the implementation of copper, lead and/or aluminum vessels helps to shield the CCD from the remaining environmental radiation.

Other sources of background are originated in the detector itself. The silicon thermal noise is the main theoretical contribution in this sense producing single-electron events. However, it may also produce two- and three-electron events as a result of the spatial pile-up of dark counts. We control this effect by cooling the detector down and reducing the exposure between reads. In addition, particles interaction in the partial charge collection layer [20] may also be a source of lowenergy noise. This layer consists of a volume in the silicon bulk where the dopant concentration is higher and, in turn, increases the recombination probability. This has been proved to be an important source of background in dark-matter experiments [21], and can be improved with a treatment to reduce the size of this layer.

The Sub-Electron-Noise Skipper-CCD Experimental Instrument (SENSEI) is the first experiment in implementing Skipper-CCDs for new physics searches. Using a prototype sensor with an active mass of about $0.1 \mathrm{~g}$ the SENSEI collaboration has been able to establish world-leading limits for dark matter in the sub-GeV mass region $[22,23]$. These limits were significantly improved with a more recent run using a scientific grade $\mathrm{CCD}$ with about a $2 \mathrm{~g}$ active mass deployed underground with $230 \mathrm{~m}$.w.e of overburden [24]. SENSEI is expected to reach an active mass of $100 \mathrm{~g}$ and run with $6000 \mathrm{~m}$.w.e of overburden, and an improved shielding by 2021 .

The Dark Matter in CCDs (DAMIC) experiment uses traditional CCDs with a resolution of about two electrons and an active mass of about $42 \mathrm{~g}$ to search for dark-matter interactions. The latest DAMIC results were obtained in a run of about 45 days with 6000 m.w.e of overburden producing leading limits in the MeV region [21, 25]. DAMIC(-M) is currently upgrading to skipper-CCDs and is expected to achieve a total active mass of $1 \mathrm{~kg}$ in the next three years [26]. In addition, the Observatory of Skipper CCDs Unveiling Recoiling Atoms (OSCURA) project, is developing a Skipper-CCD detector with a total mass of $10 \mathrm{~kg}$ to be comissioned by 2027 [27].

We show the expectations for the SENSEI final design (solid cyan line), DAMIC-M (solid red line), and OSCURA (solid blue line) in figure 6 for dark matter interacting through a light (left) and heavy (right) mediator, assuming a zero background for channels with two or more electrons. 

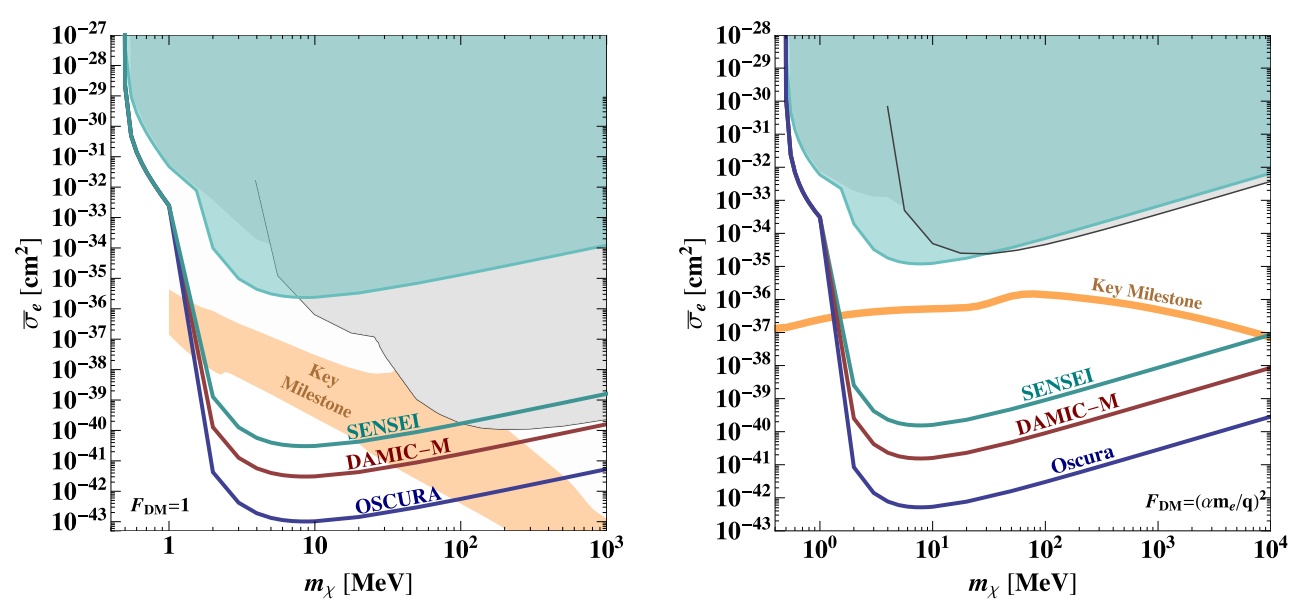

Figure 6: Projected sensitivities for SENSEI, DAMIC-M and OSCURA. The cyan shades represent the limits set by the SENSEI prototype, while the grade shades represents the limits set by DAMIC, XENON10, XENON100, XENON1T, DarkSide-50, EDELWEISS, and CDMS-HVeV. Figure adapted from [27].

Current SENSEI results are shown in a cyan shade [22-24]; DAMIC at SNOLAB [25], XENON10, XENON100, XENON1T [28-30], DarkSide-50 [31], EDELWEISS [32], and SuperCDMS [33] results are shown in a gray shade. In orange, we present the theoretical milestone scenarios, which will be broadly explore with the next generation of Skipper-CCD detectors. It is worth remarking that the SENSEI experiment with a $2 \mathrm{~g}$ skipper-CCD has set the best limits in the mass region below $10 \mathrm{MeV}$ with respect to other experiments with an active mass up to about six orders of magnitude higher. OSCURA will improve these limits in more than seven orders of magnitude.

Finally, the Dark Matter Daily Modulation Experiment $\left(\mathrm{DM}^{2}\right)$ will exploit the skipper-CCD technology to search for dark matter modulation at the south hemisphere [34]. The detector has a total active mass of about $0.1 \mathrm{~g}$ and will be deployed at $40^{\circ}$ of latitude South with an overburden of 1000 m.w.e.

Concerning neutrino detection, the Coherent Neutrino-Nucleus Interaction Experiment (CONNIE), implements scientific CCDs to detect reactor neutrino interactions in silicon. The main goal with CONNIE is to search for $\mathrm{CE} v \mathrm{NS}$ and new mediators. The nuclear energies expected in this process are about $\mathrm{keV}$, and only a fraction of this energy is translated into ionization. With a total active mass of $47.6 \mathrm{~g}$ and an anti-neutrino flux of $7.8 \times 10^{12} \mathrm{vs}^{-1} \mathrm{~cm}^{-2}$, CONNIE has set leading limits for the the $\mathrm{CE} v \mathrm{NS}$ rate bellow $0.6 \mathrm{kEV}$ and in the rates of non-standard neutrino interaction in the energy range bellow $10 \mathrm{MeV}$. The CONNIE collaboration has express interest in upgrading to a kilogram skipper-CCD detector in the next year. In addition, the Neutrino Interaction Observation with a Low Energy Threshold Array ( $v$ IOLETA) is an initiative to deploy a multi-kilogram detector to detect anti-neutrinos from a nuclear reactor. The $v$ IOLETA collaboration is projecting an unprecedented sensitivities to explore CE $v$ NS [36] by implementeing skipper-CCDs.

\section{Summary}

Skipper-CCDs are one of the most promising technologies to explore low-energy interactions: given the achieved sub-electron resolution, we can probe energy transfers as low as the silicon 
bandgap. In this work, we showed the performance of Skipper-CCD detectors and their potential to detect rare-event searches. Several are the technological challenges of scaling the active mass of a skipper-CCD from tens to achieve sub-kilogram mass up to thousands to reach multi-kilogram. The mechanical configuration to shield the CCDs from environmental radiation must allow for the cooling of the system down to about $130 \mathrm{~K}$, without introducing further noise. The electronics for signal read-out and operation setup becomes a new challenge when we increase the number of CCD to be operated in parallel. For each CCD, we normally operate four output channels and about seventy inputs to control the biases and clocks in the CCD. When scaling to about $4000 \mathrm{CCDs}$, this sums to a total of $10^{6}$ signals that need electronics that can potentially introduce new backgrounds. The challenges for a multi-kilogram Skipper-CCD system are being addressed to make possible the next generation of sub-GeV dark matter and neutrino detectors.

\section{References}

[1] G. E. Smith. Rev. Mod. Phys. 82 (2010) 2307-2312.

[2] J. Janesick. SPIE Optical Engineering Press 83 (2001) 906.

[3] W. S. Boyle and G. E. Smith, Bell Syst. Tech. 49 (1970) 587.

[4] C.J.S. Damerell, et al. Nucl. Instr. and Meth. 185 (1981) 33.

[5] S. Holland et al., IEEE Transactions on Electron Devices 50 (2003) 223-238.

[6] J. Janesick and J. Tower, Sensors 16 (2016) 688.

[7] C. Bebek et al., J. Instrum. 12 (2017) C04018.

[8] J. R. Janesick, et al. , SPIE 1242 (1990) 223-237.

[9] D. Wen, IEEE J. Solid-State Circuits 6 (1974) 410.

[10] J. Tiffenberg, Phys. Rev. Lett. 119 (2017) 6.

[11] D. Rodrigues et al., Nucl. Instrum. Meth. A 1010 (2021) 165511.

[12] The DAMIC Collaboration, Phys. Rev. B 711 (2012) 264-269

[13] The CONNIE collaboration, J. Instrum. 11 (2016) P07024.

[14] The COHERENT collaboration. Science 357 (2017) 1123-1126.

[15] R. Essig et al., J. High Energy Phys 05 (2016) 046.

[16] G. Fernandez-Moroni et al., Phys. Rev. D 91 (2015) 072001

[17] G. Fernandez-Moroni et al., Exp. Astron. 34 (2012) 43-64.

[18] M. H. White, et al.,IEEE J. Solid-State Circuits 9 (1974) 1-12.

[19] The SENSEI Collaboration, arXiv:2106.08347 (2021).

[20] G. Fernandez-Moroni et al., Phys. Rev. Applied 15 (2021) 064026.

[21] The DAMIC Collaboration, Phys. Rev. Lett. 125 (2020) 24180

[22] The SENSEI collaboration. Phys. Rev. Lett. 121 (2018) 061803.

[23] The SENSEI collaboration. Phys. Rev. Lett. 122 (2019) 161801.

[24] The SENSEI collaboration. Phys. Rev. Lett. 125 (2020) 171802.

[25] The DAMIC Collaboration, Phys. Rev. Lett. 123 (2019) 181802.

[26] The DAMIC collaboration. Nucl. Instrum. Meth. A 958 (2020) 162933.

[27] D. Baxter et al. SNOWMASS OSCURA LOI (2021).

[28] The XENON10 Collaboration, Phys. Rev. Lett. 107 (2011) 051301.

[29] The XENON100 Collaboration, Phys. Rev. D 94 (2016) 092001.

[30] R. Essig, Phys. Rev. D 96 (2017) 043017.

[31] The DarkSide Collaboration, Phys.Rev.Lett. 121 (2018) 111303

[32] The EDELWEISS Collaboration, Phys. Rev. Lett. 125 (2020) 141301.

[33] The SuperCDMS Collaboration, Phys. Rev. D 102 (2020) 091101

[34] The DM2 collaboration, Public DM2 data release (2020), zenodo.4502028

[35] The CONNIE collaboration, Phys. Rev. D 100 (2019) 092005.

[36] G. Fernandez-Moroni et al., J. High Energy Phys 03 (2021) 186. 